\title{
Revisión del género Neoquintius stat. nov., con descripción de cuatro especies nuevas y un análisis del complejo Quintius (Hemiptera: Heteroptera: Coreidae: Nematopodini)
}

\author{
A revision of the genus Neoquintius nov. status, with the description of four new species and \\ analysis of the Quintius complex (Hemiptera: Heteroptera: Coreidae: Nematopodini)
}

\author{
Harry Brailovsky* y Ernesto Barrera
}

Departamento de Zoología, Instituto de Biología, Universidad Nacional Autónoma de México. Apartado postal 70153, 04510 México, D. F., México. *Correspondencia: coreidae@ibiologia.unam.mx

\begin{abstract}
Resumen. El subgénero Neoquintius Brailovsky y Barrera (Coreidae: Nematopodini) es elevado a categoría genérica describiéndose 4 especies nuevas recolectadas en Bolivia, Panamá y Perú. Se ilustran caracteres diagnósticos del pronoto y de la cápsula genital del macho (pigóforo). Se incluye una clave para la identificación de los géneros afines a Quintius Stål y otra para las especies conocidas de Neoquintius.
\end{abstract}

Palabras clave: Insecta, Hemiptera, Heteroptera, Coreidae, Nematopidini, Neoquintius, clave, Bolivia, Panamá, Perú.

\begin{abstract}
The subgenus Neoquintius Brailovsky and Barrera (Coreidae: Nematopodini) is elevated to generic level, and 4 new species from Bolivia, Panama, and Peru are described. Diagnostic taxonomic characters of pronotum and male genital capsule (pygophore) are illustrated. An identification key to the genera closely related to Quintius Stål, as well as a key to the known species of Neoquintius are included.
\end{abstract}

Key words: Insecta, Hemiptera, Heteroptera, Coreidae, Nematopodini, Neoquintius, key, Bolivia, Panama, Peru.

\section{Introducción}

Brailovsky y Barrera (1986) describieron un nuevo subgénero (Neoquintius) dentro del género Quintius Stål (1865). La erección del mismo fue justificada al señalar inconsistencias morfológicas para ubicar 2 nuevas especies dentro de Quintius sensu-stricto (Brailovsky y Barrera, 2002, O’Shea, 1980). En este nuevo estudio se contó con un buen número de ejemplares pertenecientes a este complejo, los cuales han permitido corroborar la validez genérica de Quintius y elevar a categoría genérica Neoquintius.

Quintius queda representado por especies más esbeltas, con el artejo antenal II un poco más largo que el I, los fémures anteriores y medios de ambos sexos armados con una espina anteapical y con la tibia posterior de la hembra inerme, en tanto que en Neoquintius Brailovsky

Recibido: 18 agosto 2008; aceptado: 30 septiembre 2008 y Barrera, el artejo antenal II es más corto que el I, los fémures anteriores y medios de ambos sexos son inermes y la tibia posterior de la hembra está armada con una hilera de espinas.

Como resultado de este estudio, a las especies conocidas de Neoquintius, N. araguacitus Brailovsky y Barrera, 1986, stat. nov., y N. scenicum Brailovsky y Barrera, 1986, stat. nov., se agregan 4 nuevas especies provenientes de América Central y del Sur. Asimismo, se incluye una clave para las especies de Neoquintius y otra para la identificación de los géneros afínes a Quintius Stål.

En este artículo se emplean los siguientes acrónimos: MZUSP (Museu de Zoología, Universidade de São Paulo); IBUNAM (Colección Entomológica, Instituto de Biología, Universidad Nacional Autónoma de México); UNALM (Universidad Nacional Agraria, La Molina, Perú); y USNM (United States National Museum of Natural History, Washington, D.C.). 


\section{Clave para separar los géneros afines del complejo Quintius}

1. Artejo antenal III de longitud igual o subigual al I, delgado; espiráculo abdominal

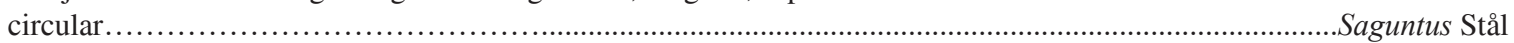

1'. Artejo antenal III mucho más corto que el I, ensanchado o delgado.....................................................2

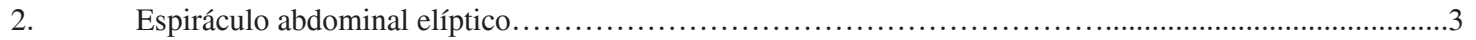

2. Espiráculo abdominal circular.......................................................................Stenoquintius Brailovsky y Barrera

3. Callo pronotal transversalmente elevado y uniformemente tuberculado; tubérculo postocular moderadamente protuberante; fémur posterior del macho más corto que la tibia posterior; fémur posterior de ambos sexos no conspicuamente ensanchados y solo ventralmente armado con una pequeña espina

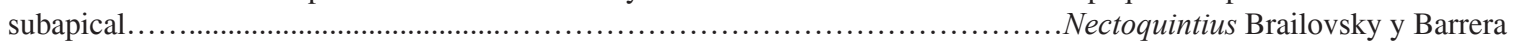

3'. Callo pronotal plano o ligeramente convexo y siempre liso, y desprovisto de tubérculos; tubérculo postocular indistinto, formando una suave curva con el ojo; fémur posterior del macho mas largo que la tibia posterior; fémur posterior de ambos sexos ensanchados y ventralmente armados con una o dos hileras de

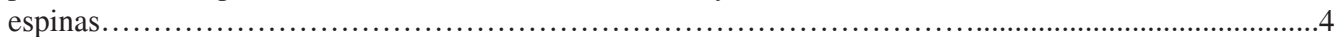

4. Fémur anterior y medio de ambos sexos armados ventralmente; tibia posterior de la hembra inerme; tibia posterior del macho provisto de una larga espina cercana al tercio medio........................................... Quintius Stål

4'. Fémur anterior y medio de ambos sexos inermes; tibia posterior de la hembra armada; tibia posterior del macho sin una larga espina cercana al tercio medio..........................................................................eoquintius Brailovsky y Barrera

\section{Descripciones}

Neoquintius bolivianus n. sp. (Figs. 1-2, 9-10, 15)

\section{Holotipo macho.}

Coloración dorsal. Cabeza. Incluyendo los artejos antenales I a IV (ápice del IV negro) anaranjado castaño pálido, con región postocular, y cuello negros. Pronoto. Anaranjado castaño pálido con puntos pardo rojizo y márgenes posterolaterales y posterior amarillo obscuro. Escutelo. Pardo rojizo brillante con márgenes laterales y ápice anaranjado castaño pálido. Hemielitro. Clavus pardo rojizo mate con la vena claval amarillo obscuro; corium pardo rojizo mate, con los márgenes costal y apical y las venas amarillo obscuro; membrana hemielitral pardo claro y con el ángulo basal pardo obscuro. Abdomen. Conexivo amarillo obscuro y con el tercio posterior del VII pardo obscuro; segmentos dorsales II a VI pardo claro y con una franja longitudinal media e irregular amarilla y el VII amarillo y con los márgenes laterales en su tercio anterior pardo claro. Coloración ventral. Cabeza. Amarillo pálido; artejos rostrales I-IV anaranjado castaño (ápice del IV negro). Tórax. Prosternón negro con el borde anterior y posterior amarillos; propleura anaranjado castaño pálido y con el área de los acetábulos negro; mesosternón y metasternón amarillo pálido; mesopleura anaranjado castaño obscuro; metapleura anaranjado castaño y con el margen posterior mayormente amarillo pálido; acetábulo medio y posterior y el lóbulo anterior y posterior del peritremo osteolar amarillo pálido. Patas. Pata anterior y media amarillo obscuro; pata posterior con las coxas, trocánteres, tibias y tarsos amarillos y con reflejos anaranjado castaño y el fémur tricolor, con la mitad basal amarillo pálido y la mitad posterior anaranjado castaño pálido con los márgenes anterior y posterior negros. Abdomen. Esternitos amarillo obscuro; cápsula genital anaranjado castaño obscuro. Estructura. Cabeza. Rostro alcanzando tercio medio del mesosternón. Pronoto. Ángulos frontales ligeramente expuestos y redondeados; ángulos humerales ligeramente agudos y salientes; bordes anterolaterales, posterolaterales y los bordes anterior y posterior lisos. Patas. Fémur anterior y medio inermes; fémur posterior dilatado, robusto y armado ventralmente con una doble hilera de espinas; tibias anterior y media inermes y surcadas; tibia posterior algo curva, surcada y armada con una serie de espinas que corren a lo largo del tercio posterior de la cara interna. Abdomen. Conexivo elevado sobre el tergum y con el borde posterior inerme. Pigóforo. Borde posteroventral con una amplia lengüeta central dirigida hacia afuera y con los ángulos laterales redondeados y suavemente convexos (Figs. 1-2).

Parámero. Figuras 9-10.

Hembra. Coloración y habitus semejante al holotipo macho. Abdomen. Segmentos conexivales VIII y IX negros y con el ángulo anterior amarillo obscuro; esternito VII anaranjado castaño obscuro, y con los márgenes posterior y pleural amarillo obscuro; segmentos dorsales VIII y IX anaranjado castaño obscuro; placas genitales anaranjado castaño obscuro.

Variación. 1. Artejos antenales II a IV anaranjado pálido. 2. Puntos del disco escutelar pardo rojizo.

Medidas. Primero el macho después la hembra. Cabeza. Longitud total: 1.44, 1.92; anchura a través de los ojos: 2.24, 2.44; distancia interocular: 1.18, 1.24; distancia 
interocelar: $0.50,0.58$; distancia preocular: 1.06, 1.28; longitud total de los artejos antenales: I, 3.28, 3.44; II, 2.84, 2.92; III, 1.72, 1.68; IV, 5.00, 4.88. Pronoto. Longitud total: 2.92, 3.44; anchura máxima a través de los ángulos humerales: 4.48, 5.16. Escutelo. Longitud: 1.84, 2.04; anchura: 1.92, 2.20. Longitud total del cuerpo: 13.95, 15.15.

Material tipo. Holotipo: macho. BoLIVIA. REGIÓN CHAPARE, Departamento Cochabamba, 400 m, 1-IX1951, Dirings (MZUSP). Paratipos. BolIVIA. REGIÓN CHAPARE. 3 hembras, Departamento Cochabamba, 400 m, 6.XI.1949 y 15.XI.1951, Dirings (MZUSP, IBUNAM).

Etimología. El nombre específico hace referencia a su distribución en Bolivia.

\section{Comentarios taxonómicos}

Especie cercana a N. scenicum Brailovsky y Barrera (1986), ambas poseen el fémur posterior tricolor, con la mitad basal amarilla y el resto negro y con un amplio anillo central anaranjado obscuro. En $N$. scenicum el margen apical del corium y cercano al ángulo apical posee una mancha amarilla (ausente en $N$. bolivianus), la mesopleura muestra una franja longitudinal negra que corre por encima de los acetábulos (ausente en $N$. bolivianus), y los ángulos humerales son redondeados y no expuestos, mientras que en $N$. bolivianus son agudos y salientes (Fig. 15).

Neoquintius chaparenus n. sp. (Figs. 3-4, 12-13)

\section{Holotipo macho.}

Coloración dorsal. Cabeza. Incluyendo artejos antenales I a IV anaranjado castaño brillante, con región postocular y cuello negros. Pronoto. Anaranjado castaño con los puntos pardo rojizo y los bordes anterior, posterior y posterolaterales anaranjado obscuro; espina de los ángulos humerales negra. Escutelo. Pardo rojizo brillante y con márgenes laterales y ápice anaranjado amarillento. Hemielitro. Clavus pardo rojizo mate con franja longitudinal amarilla situada por encima de la vena claval; corium pardo rojizo mate con los bordes costal y apical y las venas amarillo obscuro; membrana hemielitral pardo obscuro. Abdomen. Segmentos conexivales III a VI amarillos y el VII pardo rojizo; segmentos dorsales negros y con las áreas cicatriciales IV-V y V-VI amarillas. Coloración ventral. Cabeza. Incluyendo los artejos rostrales (ápice del IV negro) anaranjado castaño brillante. Tórax. Prosternón pardo obscuro; mesosternón y metasternón pardo obscuro y con una franja longitudinal media amarillo obscuro; propleura anaranjado castaño con los puntos y el área anexa a los acetábulos pardo rojizo; mesopleura y metapleura anaranjado castaño brillante, con los puntos y el margen superior de la mesopleura pardo rojizo; lóbulos anterior y posterior del peritremo osteolar amarillo obscuro. Patas. Pata anterior y media anaranjado castaño brillante; pata posterior con coxa anaranjado brillante y la cara externa mayormente negra; trocánter amarillo; fémur anaranjado obscuro con los gránulos y las espinas ventrales pardo rojizo; tibia y tarsos amarillo obscuro. Abdomen. Esternitos anaranjado obscuro con el margen pleural amarillo y el margen posterior del VII negro; pigoforo anaranjado obscuro, con una mancha central y los márgenes laterales pardo rojizo. Estructura. Cabeza. Rostro alcanzando tercio medio del mesosternón. Pronoto. Ángulos frontales ligeramente expuestos y redondeados; ángulos humerales conspicuamente desarrollados en una espina larga y aguda, dirigida hacia fuera y ligeramente hacia delante (Fig. 13). Patas y abdomen semejantes a $N$. bolivianus. Pigóforo. Borde posteroventral con una lengüeta mesial orientada hacia fuera (Figs. 3-4). Parámero. Figura 12.

Hembra. Desconocida.

Medidas. Macho. Cabeza. Longitud total 1.68; anchura a través de los ojos: 2.24; distancia interocular: 1.22; distancia interocelar: 0.52 ; distancia preocular: 1.28; longitud de los artejos antenales: I, 3.00; II, 2.80; III, 1.60; IV, 4.36. Pronoto. Longitud total: 3.20; anchura máxima a través de los ángulos humerales: 5.20. Escutelo. Longitud: 1.92; anchura: 2.16. Longitud total del cuerpo: 14.42 .

Material tipo. Holotipo: macho. BoLIVIA. REGIÓN CHAPARE, Departamento Cochabamba, 400 m, 10.V.1949, Dirings (MZUSP).

Etimología. Nombre específico alusivo a su distribución en la Región Chapare de Bolivia.

\section{Comentarios taxonómicos}

Esta especie se reconoce por tener los ángulos humerales del pronoto proyectados en una larga y aguda espina, dirigida hacia fuera y algo hacia delante (Fig. 13), y los fémures posteriores unicolor, de un tinte anaranjado obscuro. En N. araguacitus Brailovsky y Barrera (1986) los fémures posteriores son enteramente amarillo o anaranjado pálido y los ángulos humerales obtusos. En $N$. bolivianus descrito en párrafos anteriores y en N. scenicum Brailovsky y Barrera (1986) los fémures posteriores son tricolores.

Neoquintius panamensis n. sp. (Figs. 5-7)

Holotipo macho.

Coloración dorsal. Cabeza. Incluyendo los artejos 
antenales I a IV amarillo anaranjado y con la región postocular y el cuello negros. Pronoto. Anaranjado castaño obscuro con los puntos pardo rojizo, ángulos humerales negros y bordes anterior, posterior y posterolaterales amarillo obscuro. Escutelo. Pardo rojizo con márgenes laterales y ápice amarillos. Hemielitro. Clavus pardo rojizo mate, con franja longitudinal amarilla que corre a lo largo de la vena claval: corium pardo rojizo mate con el margen costal y apical, las venas y una mancha irregular por encima del margen apical amarillo; membrana hemielitral pardo obscuro. Abdomen. Segmentos conexivales II a VI anaranjados con las uniones intersegmentales pardo rojizo y el VII pardo rojizo y con el ángulo anterior amarillo; segmentos dorsales pardos con las cicatrices odoríferas IV-V y V-VI amarillas. Coloración ventral. Cabeza. Incluyendo los artejos rostrales anaranjado castaño (ápice del IV negro). Tórax. Prosternón con el borde anterior y posterior amarillos; mesosternón negro y con franja longitudinal media amarilla; metasternón pardo obscuro y con una mancha mesial anaranjado obscuro; acetábulos pardo anaranjado obscuro con puntos pardo rojizo; propleura amarillo sucio y con puntos pardo rojizo; mesopleura anaranjado obscuro con el margen superior mayormente negro; metapleura incluyendo margen posterior anaranjado obscuro; peritremo osteolar amarillo. Patas. Patas anterior y media anaranjado castaño brillante, excepto coxas mayormente negras; pata posterior con coxas negras, trocánter amarillo obscuro y con mancha discoidal apical negra, fémur anaranjado obscuro y tibias y tarsos amarillos. Abdomen. Esternitos abdominales III a VI anaranjado obscuro con margen pleural amarillo y VII anaranjado obscuro con margen posterior negro y margen pleural amarillo sucio; pigóforo anaranjado obscuro. Estructura. Cabeza. Rostro alcanzando tercio medio del mesosternón. Pronoto. Ángulos frontales ligeramente expuestos y redondeados; ángulos humerales conspicuamente largos, agudos y dirigidos hacia fuera y algo hacia atrás. Patas. Similares a las descritas para N. bolivianus. Abdomen. Connexivo elevado sobre el tergum y con borde posterior inerme. Pigóforo. Borde posteroventral con amplia lengüeta mesial, dirigida hacia fuera y con ángulos laterales redondeados (Figs. 5-6). Parámero. Figura 7.

Hembra. Coloración y habitus semejante al holotipo macho. Patas. Coxas anteriores y medias amarillas. Abdomen. Segmentos conexivales VII a IX anaranjado obscuro con borde posterior pardo; segmentos dorsales VIII y IX pardo rojizo; placas genitales anaranjado obscuro; esternitos III a VII anaranjado obscuro y con margen pleural amarillo.

Medidas. Primero el macho después la hembra. Cabeza. Longitud total: 1.92, 1.90; anchura a través de los ojos: 2.48, 2.52; distancia interocular: 1.32, 1.46; distancia interocelar: 0.54, 0.56; distancia preocular: 1.20, 1.40; longitud total de los artejos antenales: I, 3.32, 3.44; II, 3.08, 3.08; III, 1.60, 1.60; IV, 5.08, ausente. Pronoto. Longitud total: 3.44, 3.68; anchura máxima a través de los ángulos humerales: 6.04, 6.52. Escutelo. Longitud: 1.92, 2.08; anchura: 2.20, 2.52. Longitud total del cuerpo: 15.47, 15.71 .

Material tipo. Holotipo: macho. PANAMÁ. Gamboa, 1725.VIII.1986, C. Riley Nelson (Malaise trap) (UNAM). Paratipo. Panamá. 1 hembra, Pecora, 6.II.1945, H. H. Stage (USNM).

Etimología. El nombre específico se refiere a su distribución en Panamá.

\section{Comentarios taxonómicos}

Al igual que $N$. chaparenus descrito de Bolivia tiene los fémures unicolores y los ángulos humerales del pronoto desarrollados en una larga y aguda espina dirigida hacia afuera que en $N$. chaparenus se curva hacia afuera y un poco hacia delante (Fig. 13) y en N. panamensis hacia afuera y atrás.

El corium de $N$. panamensis posee una mancha alargada e irregular de color amarillo sobre el margen apical, ausente en $N$. chaparenus.

Neoquintius peruvianus n. sp. (Figs. 8, 11, 14)

\section{Holotipo macho.}

Coloración dorsal. Cabeza. Amarilla con reflejos anaranjado castaño y con región postocular y cuello negros; artejo antenal I amarillo y artejos II a IV anaranjados. Pronoto. Amarillo anaranjado obscuro con puntos pardo rojizo y collar, ángulos frontales y bordes posterior y posterolaterales amarillo más pálidos. Escutelo. Anaranjado castaño, con márgenes laterales y ápice amarillos. Hemielitro. Clavus pardo rojizo mate con franja longitudinal amarilla que corre a lo largo de la vena claval; corium pardo rojizo mate con margen costal y apical y venas amarillas; membrana hemielitral pardo obscuro. Abdomen. Segmentos conexivales III a VI amarillos y VII pardo obscuro con borde anterior amarillo; segmentos dorsales anaranjado castaño pálido con áreas cicatriciales IV-V y V-VI amarillas. Coloración ventral. Cabeza. Incluyendo los artejos rostrales I-IV (ápice del IV negro) amarillos y con reflejos anaranjado castaño. Tórax. Prosternón negro y con margen posterior amarillo; mesosternón, metasternón, lóbulos anterior y posterior del peritremo osteolar y acetábulos de los tres pares de patas amarillo pálido; propleura amarillo obscuro con puntos pardo rojizo y una franja negra que corre por encima de los acetábulos; mesopleura amarillo obscuro con puntos 
pardo rojizo; metapleura anaranjado castaño brillante y con margen posterior amarillo pálido. Patas. Patas anterior y media con coxas y trocanteres amarillo pálido y resto amarillo obscuro; pata posterior con coxas, trocanteres, tibias y tarsos amarillo obscuro y fémur tricolor, con la mitad basal anaranjado castaño brillante y mitad apical negra y con un anillo central ensanchado e incompleto de color anaranjado castaño mate. Abdomen. Esternitos III a VI amarillo obscuro con reflejos irregulares pardos y VII amarillo obscuro con el margen posterior y una mancha cercana a la línea media pardo anaranjada; pigóforo anaranjado castaño obscuro con reflejos pardo anaranjado. Estructura. Cabeza. Rostro alcanzando el tercio medio del mesosternón. Pronoto. Ángulos frontales ligeramente expuestos y redondeados; ángulos humerales truncados y no expuestos (Fig. 14). Patas, abdomen y pigóforo similares a lo descrito para N. bolivianus. Parámero. Figuras 8, 11. Hembra. Se desconoce.

Medidas. Macho. Cabeza. Longitud total: 1.60; anchura a través de los ojos: 2.08; distancia interocular: 1.02; distancia interocelar: 0.42 ; distancia preocular: 1.02 ; longitud total de los artejos antenales: I, 2.98; II, 2.62; III, 1.64; IV, 4.04. Pronoto. Longitud total: 2.58; anchura máxima a través de los ángulos humerales: 3.92. Escutelo. Longitud: 1.62; anchura: 1.80. Longitud total del cuerpo: 12.50 .

Material tipo. Holotipo: macho. PERú. San Martín Tarapoto, río Shilcayo, 26.X.2000, S. Schutler (UNALM).

Etimología. El nombre específico hace referencia a su distribución en Perú.

\section{Comentarios taxonómicos}

Neoquintius peruvianus al igual que $N$. bolivianus y $N$. chaparenus carecen de una mancha amarilla que corre sobre el margen apical del corium y que esta presente en $N$. panamensis. En N. peruvianus y $N$. bolivianus el fémur posterior es tricolor mientras que en $N$. chaparenus unicolor. Los ángulos humerales del pronoto de $N$. bolivianus están ligeramente expuestos y son subagudos (Fig. 15) y en $N$. peruvianus son truncados y no expuestos (Fig. 14).

\section{Clave para separar las especies de Neoquintius}

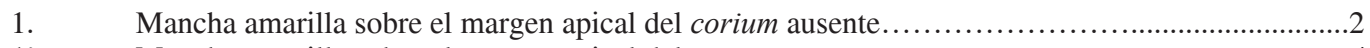

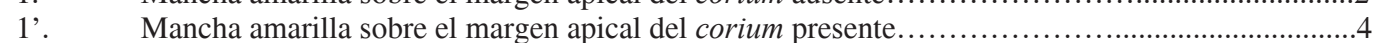

2. Fémur posterior unicolor; ángulos humerales del pronoto proyectados en una larga y aguda espina dirigida hacia fuera y ligeramente hacia delante (Fig. 13)...............

$2^{\prime}$ Fémur posterior tricolor; ángulos humerales del pronoto subagudos o truncados, y nunca proyectados en una espina larga y

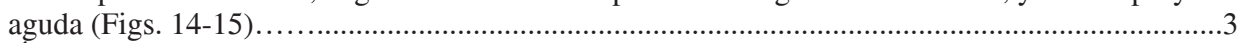

3. Ángulos humerales del pronoto truncados y no expuestos; mitad apical del fémur posterior negro y con un anillo mesíal incompleto anaranjado obscuro.................................................................................. peruvianus $\mathrm{n} . \mathrm{sp}$.

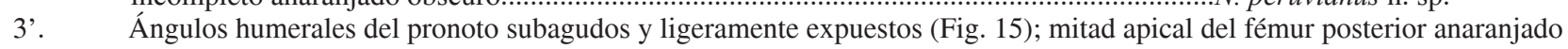
castaño pálido y con el margen anterior y posterior negros............................... bolivianus n. sp.

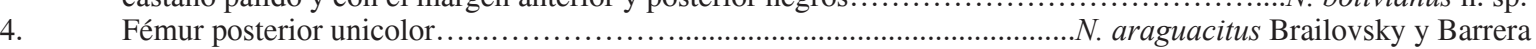

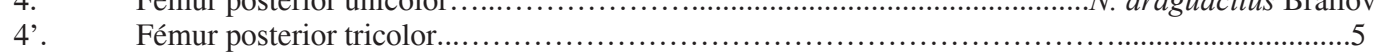

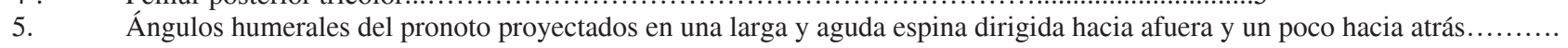

5.

5'. Ángulos humerales del pronoto redondeados y no expuestos.

.N. scenicum Brailovsky y Barrera

\section{Agradecimientos}

A los siguientes colegas e instituciones por haber facilitado en préstamo gran parte del material aquí estudiado: Sonia Casari (MZUSP), Clorinda Vergara (UNALM) y Thomas J. Henry (USNM). A Luis García Prieto (UNAM), por el apoyo en computación y a Elvia Esparza (UNAM) y Albino Luna (UNAM), por los dibujos en vista dorsal de las especies estudiadas. Especial agradecimiento a los arbitros anónimos por el tiempo empleado para mejorar el texto.

\section{Literatura citada}

Brailovsky, H., y E. Barrera. 1986 (1985). El género Quintius Stål con descripción de un subgénero nuevo y tres especies nuevas (Hemiptera-Heteroptera-Coreidae-Nematopodini). Anales del Instituto de Biología, Universidad Nacional Autónoma de México, Serie Zoología 56:437-452.

Brailovsky, H. y E. Barrera. 2002. New genera and new species of Neotropical Nematopodini (Hemiptera: Heteroptera: Coreidae: Coreinae). Pan-Pacific Entomologist 78:265-275.

O'Shea, R. 1980. A Generic Revision of the Nematopodini (Heteroptera: Coreidae: Coreinae). Studies on Neotropical Fauna and Environment 15:197-225. 

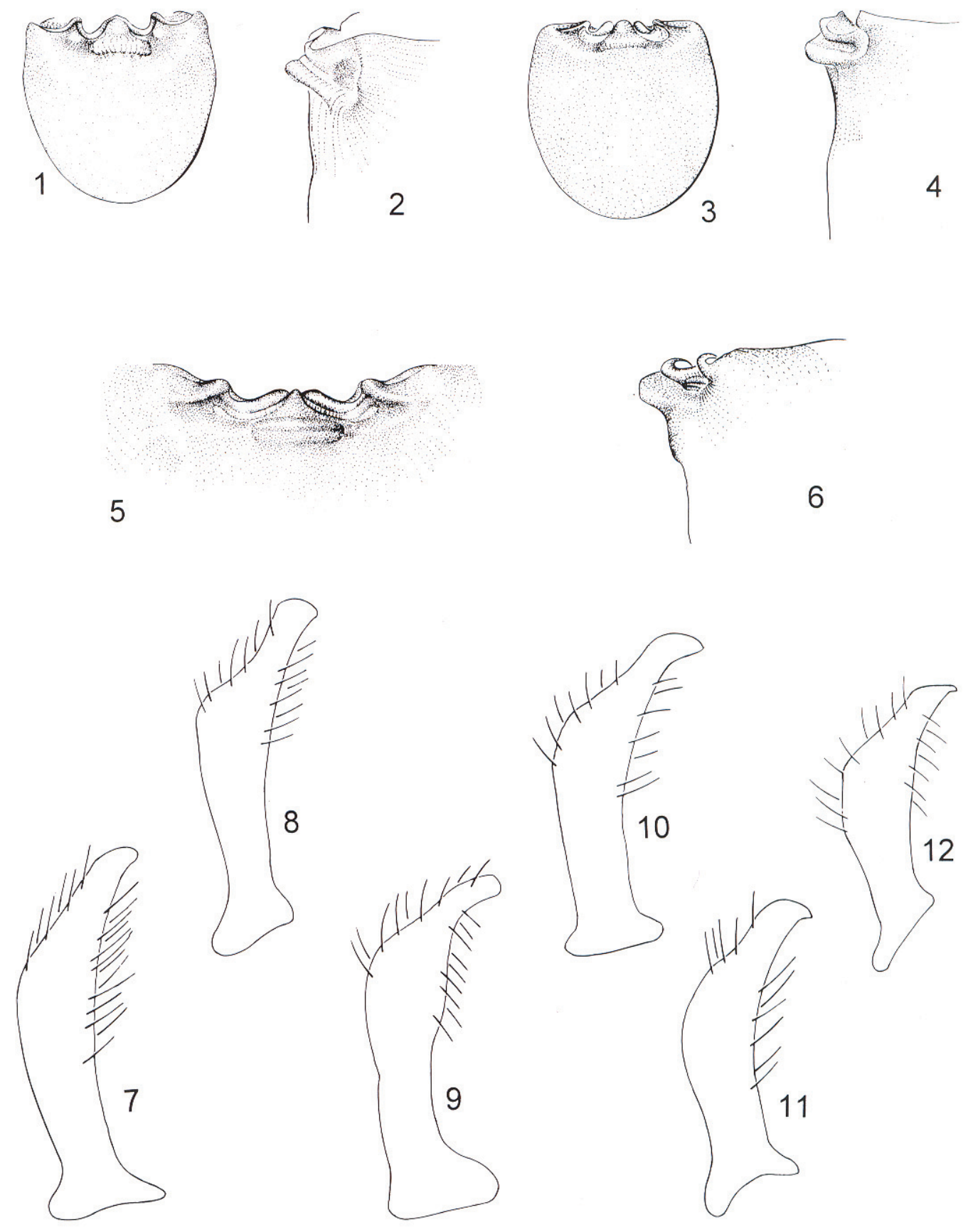

Figuras 1-12. Neoquintius spp. 1-6. Pigóforo. 1-2, N. bolivianus n. sp. 1, vista caudal; 2, vista lateral. 3-4. N. chaparenus n. sp. 3, vista caudal; 4, vista lateral. 5-6. N. panamensis n. sp.; 5, vista caudal; 6, vista lateral; 7-12. Parámero. 7. N. panamensis n. sp. 8-11. $N$. peruvianus $\mathrm{n}$. sp. 9-10. $N$. bolivianus $\mathrm{n}$. sp. 12. $N$. chaparenus $\mathrm{n}$. sp. 

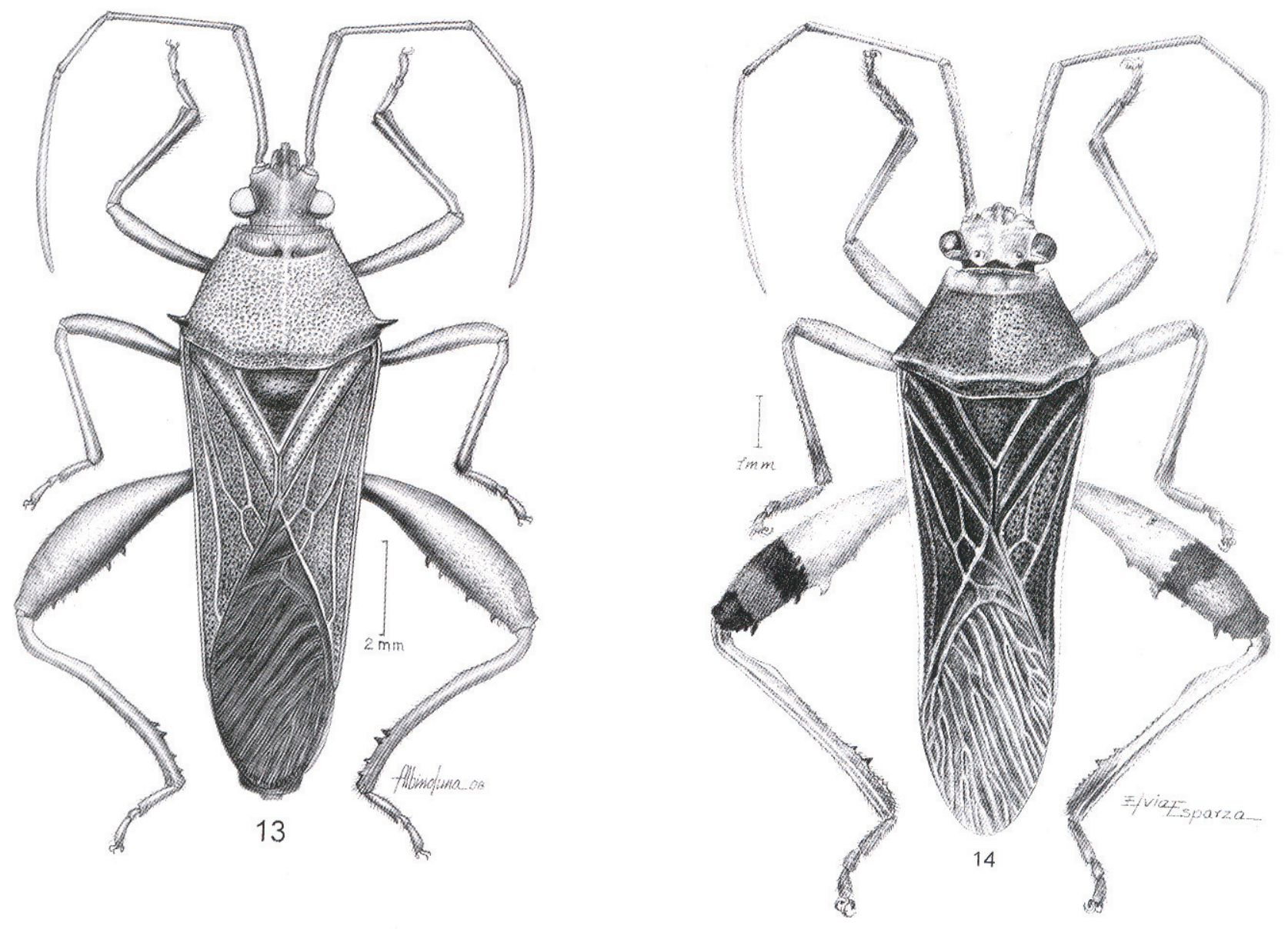

Figura 13. Vista dorsal de Neoquintius chaparenus n. sp.

Figura 14. Vista dorsal de Neoquintius peruvianus n. sp. 


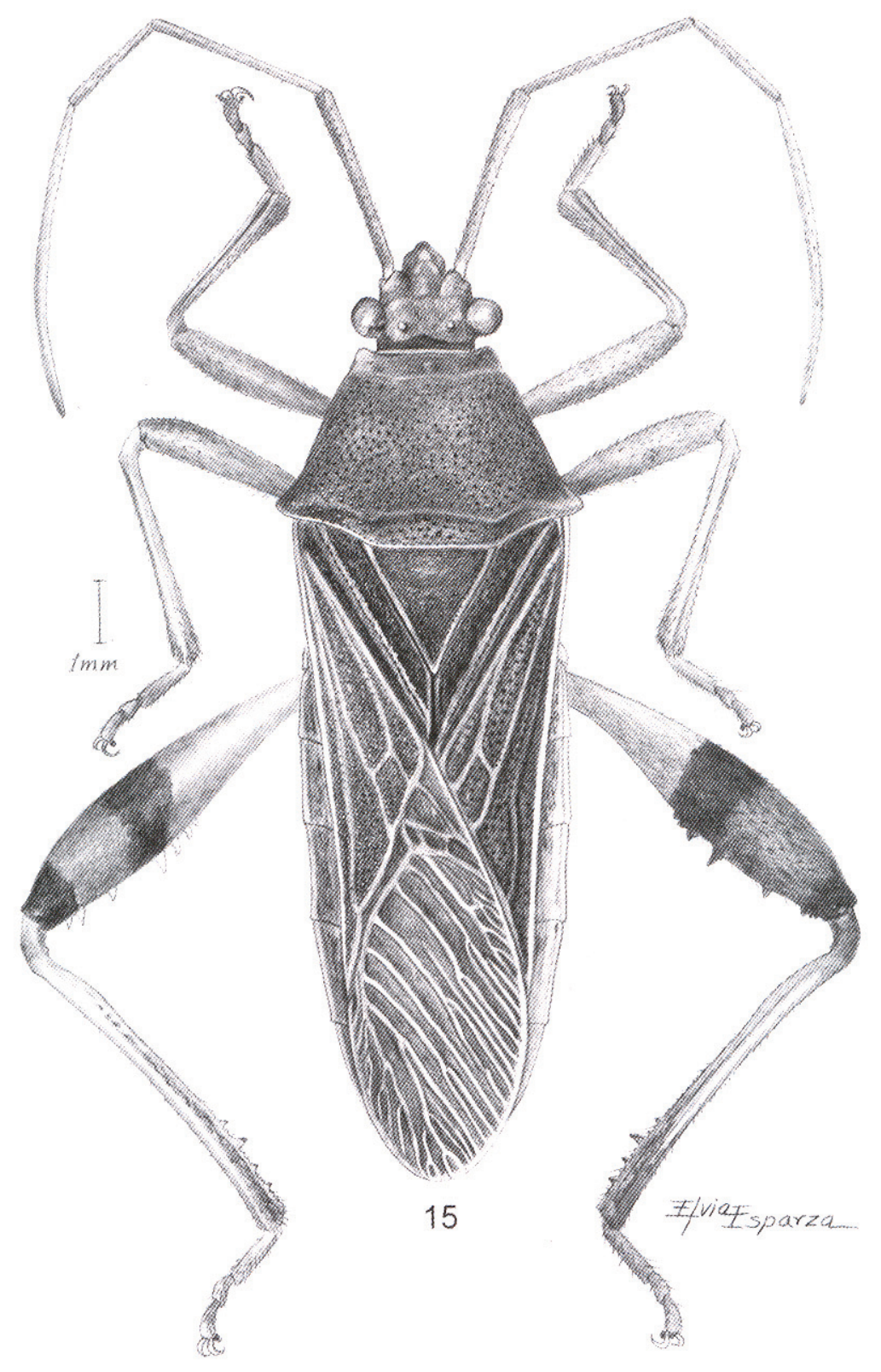

Figura 15. Vista dorsal de Neoquintius bolivianus n. sp. 